\title{
Near-field frequency attenuation of vibrations from blasting using a full-field solution
}

\author{
S. Gómez, J. A. Sanchidrián, P. Segarra \\ Universidad Politécnica de Madrid-ETSI Minas y Energía, Madrid, Spain
}

\begin{abstract}
This study analyses the frequency content of vibrations from blasting in the near field using a vibration prediction model called full-field solution (FFS). For that purpose, the frequency content of recorded signals and synthetic waveforms obtained through the FFS using dominant and mean frequencies have been compared and correlated with the geometrical distance to the blastholes. The study shows that the model overestimates the dominant and mean frequencies in the entire frequency domain, which may require tailoring the source function to a lower frequency content. However, it attenuates the frequency content as distance increases. Additionally, the study shows that the mean frequencies may be used as a better estimator of the frequency content than the dominant frequencies.
\end{abstract}

\section{INTRODUCTION}

It is common in the mining industry to measure the frequency content and peak vibration levels from blasting, to control and prevent any potential damage to structures at some distance from the blast. To that end, there is a multitude of criteria and standards, like the SpanishUNE 22.381-93 [1]. However, it is less common to perform these measurements in the vicinity of the blasthole, at distances in the so-called near-field range, where frequency content is more difficult to predict.

Typically, the parameter used to characterize the frequency content of a signal is the dominant frequency. There are many scientific publications that use empirical models to obtain them as a function of the maximum explosive charge per delay, the distance between blasting source and measuring point, peak particle velocity, longitudinal and shear wave velocities in the rock, the relative elevation, among other empirical coefficients [9]. However, it is preferable to resort to analytical or numerical vibration prediction models with which the full-waveform and therefore its corresponding frequency spectrum may be obtained.

Frequency attenuation of seismic waves from blasting occurs in a way that the highfrequency components of an incident wave are more attenuated during wave propagation than the low-frequency components. In that sense, the frequency spectrum undergoes a downshift in frequency as the wave propagates, since the high-frequency part of the spectrum decreases faster than the low-frequency part [19]. Since the power spectrum shifts toward low frequency-components, we consider the mean frequency (or the spectral centroid of the frequency spectrum)as a better estimator of the frequency content than the dominant frequency or frequency at which the spectral amplitude is maximum. The main 
reason is that, even though most of the energy in a power spectrum may often be concentrated around the dominant frequency, there are many situations in which it is not. An example of this is when two or more frequencies in the power spectrum present very similar amplitudes. This may be an issue when characterizing the frequency content of a signal through the dominant frequency.

The two aims of this study are: (i) to analyse the effect of distance in the frequency content of waveforms predicted by an analytical vibration prediction model called full-field solution (FFS) and thus show the validity of this approach to describe quantitatively vibrations in the near field and (ii) describe the frequency content of vibrations with a single frequency value that is sensitive to the effect of distances; this paper shows that mean frequency is more suitable for characterization of the frequency content of a seismic signal from blasting than the commonly sued dominant frequencies.

The full-field solution is a semi-analytical and physically sound vibration prediction model whose origin dates back to the mid-1980s and early 1990s [16, 20]. It has had a certain impact in the last 15 years $[3,4,5,8]$, but it is still far from having the same popularity as the well-known Holmberg-Persson model and its derivatives[11, 12, 13]. This is mainly due to the full-field solution calculation complexity, implementation difficulties, and longer calculation times.

As rocks do not behave as purely elastic solids, a viscoelastic attenuation model must be incorporated into the full-field solution. Kjartansson's constant- $Q$ (CQ) model [14]is commonly used because it provides a realistic description of the intrinsic attenuation in dry crystalline rock [2] and it is a single-parameter dependent model.

\section{SITE AND TESTS}

A total of twelve blasts were carried out in El Aljibe, a quarry located in Almonacid de Toledo, Spain. The deposit is in a ductile shear metamorphic zone (the Toledo mylonite belt) that developed during the final tectonic event of the Hercynian Orogeny [10]. Figure 1 shows a map of Spain and an aerial view of the open pit. The quarry mylonite mines are primarily intended for ballast and other construction aggregates. The blasts were located in the southwest part of the main pit at the deepest level.The mechanical properties of the rock have been taken from [8]. They are summarized in Table 1.

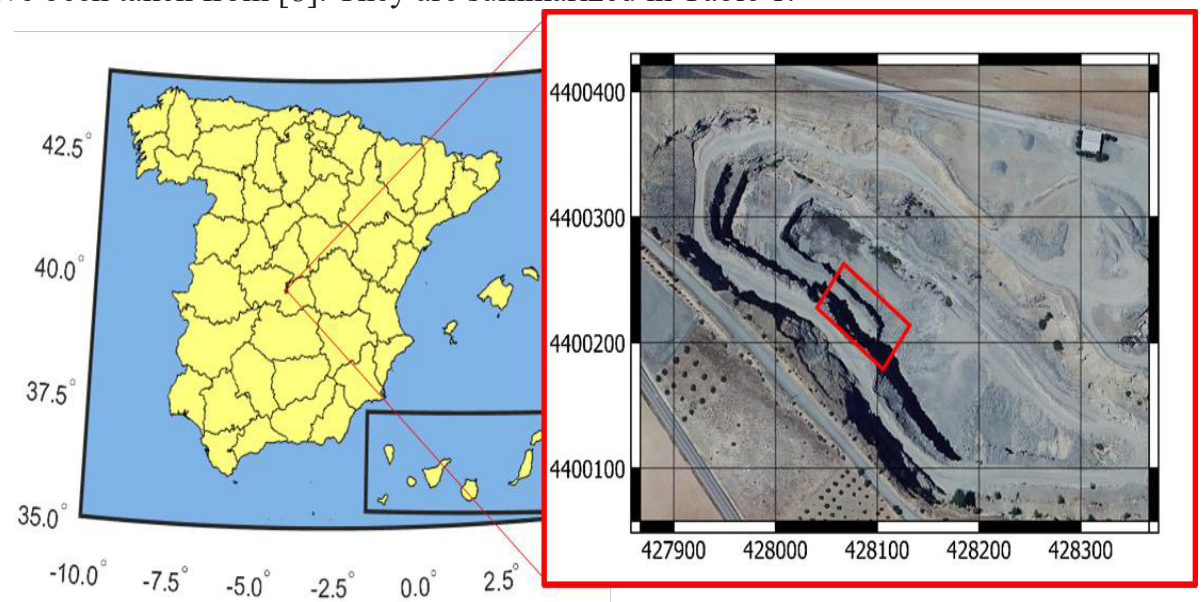

Fig. 1. Location of El Aljibe quarry. Datum: ETRS 89 / UTM Zone 30N (EPSG 25830). Equidistant conic projection. 
Table 1. Mechanical properties of the rock mass ${ }^{1}$.

\begin{tabular}{ccccccc}
\hline$V_{p}(\mathrm{~m} / \mathrm{s})$ & $V_{s}(\mathrm{~m} / \mathrm{s})$ & $\rho\left(\mathrm{kg} / \mathrm{m}^{3}\right)$ & $v$ & $E(\mathrm{GPa})$ & $U C S(\mathrm{MPa})$ & $T S(\mathrm{MPa})$ \\
\hline 5844 & 3300.4 & 2721.2 & 0.24 & 74.76 & 171.21 & 18.21 \\
\hline
\end{tabular}

${ }^{1} V_{p}$ and $V_{s}:$ p- and s-wave velocities; $\rho$ : density, $v$ :Poison's ratio;E: Young's modulus; UCS:uniaxial compressive strength;TS: tensile strength.

Each blast consisted of one row composed of seven $89 \mathrm{~mm}$ diameter inclined blastholes, each one delayed 4 or $23 \mathrm{~ms}$ depending on the blasting number. The shots were bottom initiated with $400 \mathrm{~g}$ boosters and electronic detonators. Blastholenumber 7 (BH\#7) was always drilled in the northern part of the bench and the first to be fired. Only blasts with a 23 msdelay between holes have been considered in this study to distinguish arrivals corresponding to each hole. They are called B3, B6, B7, and B9.

Seismic records were obtained experimentally using fiveshock ceramic-shear accelerometers and a high-rank geophone, which were arranged in a vertical orientation and located at distances from the blastholes from 3.3 to $29.1 \mathrm{~m}$. Measurement ranges were $10000 \mathrm{~g}$ (A1), $5000 \mathrm{~g} \mathrm{(A2),} \mathrm{and} 500 \mathrm{~g}$ (A3) for the accelerometers and $2 \mathrm{~m} / \mathrm{s}$ (G1) for the geophone.Sensors were placed in pairs $(\mathrm{A} 1 / \mathrm{G} 1, \mathrm{~A} 2 / \mathrm{A} 3, \mathrm{~A} 2 \mathrm{~b} / \mathrm{A} 3 \mathrm{~b})$ at the bottom of $6 \mathrm{~m}$ boreholes behind the blasts to reduce as far as possible the influence of free faces. The sensors weregrouted to the hole walls ensuring a firm attachment of the sensors to the rock mass.Figure 2 shows a sketch of the blasts and sensors' layout.The data acquisition system was composed of two DATATRAP II manufactured by MREL; a sampling rate of $10 \mathrm{MHz}$ was used.

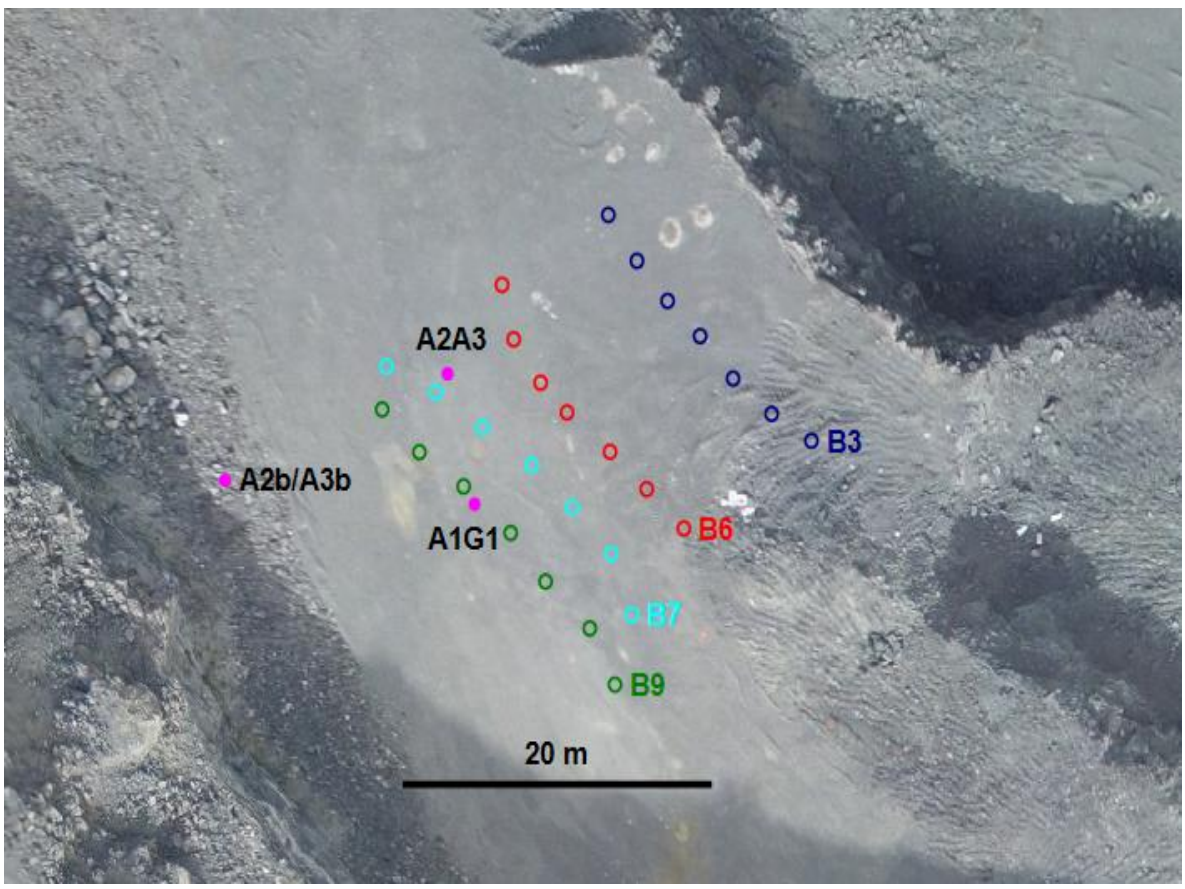

Fig. 2. Blasts and sensors layout.Solid circles: sensor holes; empty circles: blasthole collars. 


\section{Methods}

\subsection{Full-field solution (FFS)}

The FFS predicts the behaviour of an isotropic medium upon detonation of a cylindrical explosive charge of finite length. The displacement field from an element of charge can be represented by three displacement potentials, one of them corresponding to a longitudinal or primary wave and the other two to transverse or shear waves [16]. To model the detonation, elements of small length are superimposed in a way that they apply a load to the blasthole wall one after the other with a time difference equal to the travel time of the detonation from one element to the other Blair [4].

The mathematical formulation of the full-field solution has already been widely studied by other authors. In this study, we use the one developed by [8].The fundamental equation of the model is:

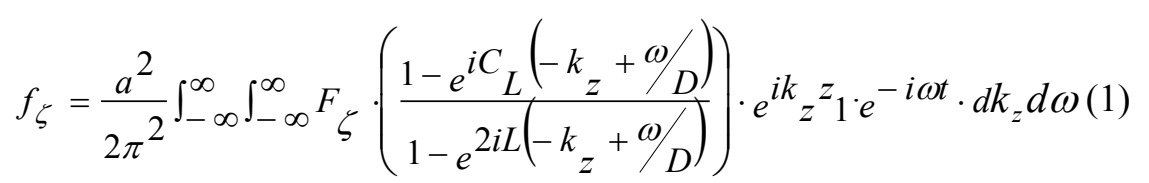

where $f_{\xi}$ is the $\zeta$-component of any field $f$ (displacement, velocity, etc.) from the detonation of the entire column of explosive at a certain location, $\zeta$ denotes the corresponding direction i.e., radial $(r)$ or axial $(\mathrm{z}), a$ is the blasthole radius $(\mathrm{m}), F_{\zeta}$ is the wave number-frequency transform of the $\zeta$-component field. It depends on the radial distance to the monitoring point ( $r$ in meters), the axial wave number, $k_{z}(\mathrm{rad} / \mathrm{m})$, and the angular frequency $\omega(\mathrm{rad} / \mathrm{s})$; $L$ is the half-length of an element of explosive $(\mathrm{m}), C_{L}$ is the length of the explosive column (m), $D$ is the detonation velocity $(\mathrm{m} / \mathrm{s}), z_{l}$ is the axial coordinate of the monitoring point as if it were seen from the centre of the first element that detonates and the time in seconds.

$F_{\zeta}$ consists of three terms: the spectral response of the source function, a sinusoidal term, and a directional term [4]. Perhaps the most important here is the first one because vibration levels and frequency content depend largely on it. In this work, we use a pressure-time history obtained in the context of a wider project by numerically modelling the detonation $[6,7]$. The directional term includes the attenuation model in form of two-phase velocity functions dependent on angular frequency, one for each wave velocity. To take into account the explosive-to-rock shock matching, the peak pressure applied in the blasthole wall is determined by the intersection of the direct Hugoniot of the rock with the reflected Hugoniot of the detonation products at the Chapman-Jouget point. Some numerical techniques must be used to solve Eq. (1). The problem is divided into two parts, one for each integral. The inner integral is solved numerically by using an adaptive quadrature method; the outer integral is solved using an Inverse Fast Fourier Transform algorithm. A more detailed description of the essence of numerical computations may be seen in [8].

In this case, as blastholes and sensors are not aligned (since holes were drilled at an inclination), the two components of any field (radial and axial) are projected in the vertical direction of the sensor. Table 2 shows the distances of the centres of mass of the blast holes to the sensors. For this purpose, signals recorded are labelled AxBy and GxBy for accelerometers and geophones, respectively, " $x$ " being the sensor number and " $y$ " the blasting number.

Table 2. Geometrical distance from the blasthole'scenter of mass to the monitoring points in $(\mathrm{m})$.

\begin{tabular}{llllllll}
\hline & BH\#1 & BH\#2 & BH\#3 & BH\#4 & BH\#5 & BH\#6 & BH\#7 \\
\hline A2B & 25.31 & 22.92 & 20.23 & 18.27 & 16.48 & 15.83 & 16.03 \\
\cline { 2 - 7 }
\end{tabular}




\begin{tabular}{llllllll}
\hline $\begin{array}{l}3 \\
\text { A3B } \\
3\end{array}$ & & & & & & & \\
\hline $\begin{array}{l}\text { A2B } \\
6\end{array}$ & 18.2 & 15.45 & 12.42 & 9.22 & 7.5 & 5.97 & 7.66 \\
\hline $\begin{array}{l}\text { A2B } \\
7\end{array}$ & & & & & & & \\
A3B & 28.9 & 26.77 & 24.92 & 22.16 & 19.14 & 16.84 & 16.22 \\
7 & & & & & & & \\
\hline $\begin{array}{l}\text { A3B } \\
9\end{array}$ & 29.06 & 26.41 & 23.16 & 20.1 & 16.78 & 14.31 & 13.32 \\
\hline $\begin{array}{l}\text { A1B } \\
9\end{array}$ & & & & & & & \\
$\begin{array}{l}\text { G1B } \\
9\end{array}$ & - & & 7.54 & 4.44 & 3.32 & 4.88 & 8.77 \\
\hline
\end{tabular}

The CQ attenuation model is incorporated to replicate the intrinsic attenuation of seismic waves as they travel through the rock mass. We do not know the $Q$-value to be used in the full-field solution beforehand. Consequently, a calibration of $Q$ with the distance to the source was made taking advantage that peak vibration levels (peak particle accelerations and velocities) are proportional to the $Q$-value. Once the $Q$-law is obtained, we may obtain the full waveforms at the same locations where sensors were placed. This allows calculating the frequency spectrum of the signals obtained from the full-field solution. In this work, we use the $Q$-law obtained by Gómez et al.

As an example, Figure 3 shows what the peak particle velocity radiation pattern for a 3 m-blasthole, $Q=15$ and $D=4500 \mathrm{~m} /$ supwards looks like. 


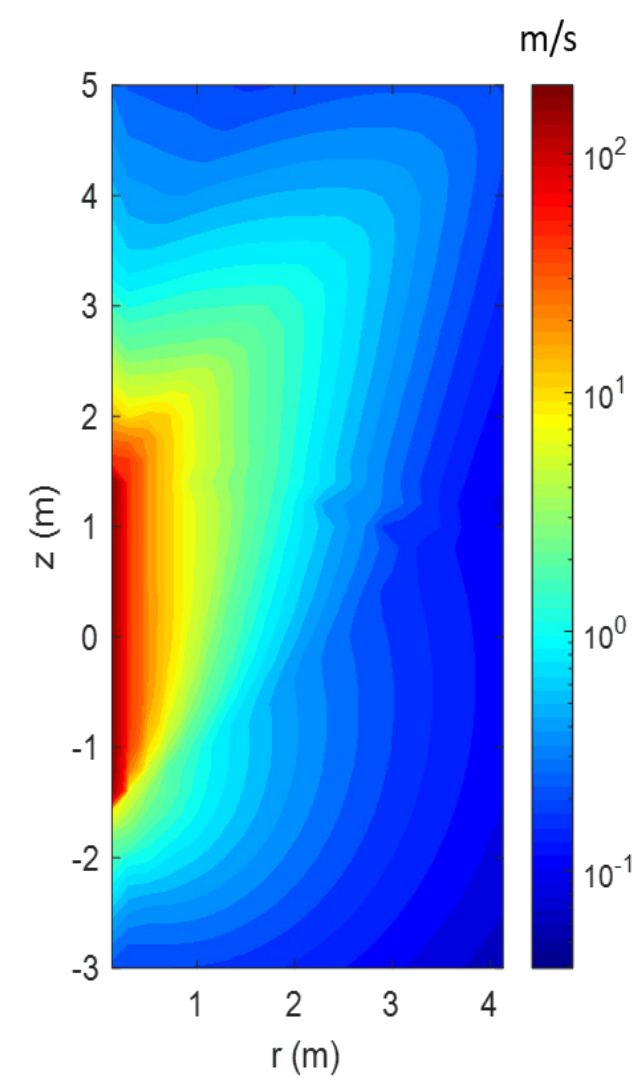

Fig. 3.Peak particle velocityradiation pattern of a $3 \mathrm{~m}$ blasthole.

\subsection{Frequency Content Calculation}

The recorded and modelled signals were passed through a 23 ms-bandwidth rectangular mobile window in order to isolate the frequency content corresponding to every single blasthole. Figure 4 shows an example of a signal from sensor A2 in blast B3 (A2B3). 


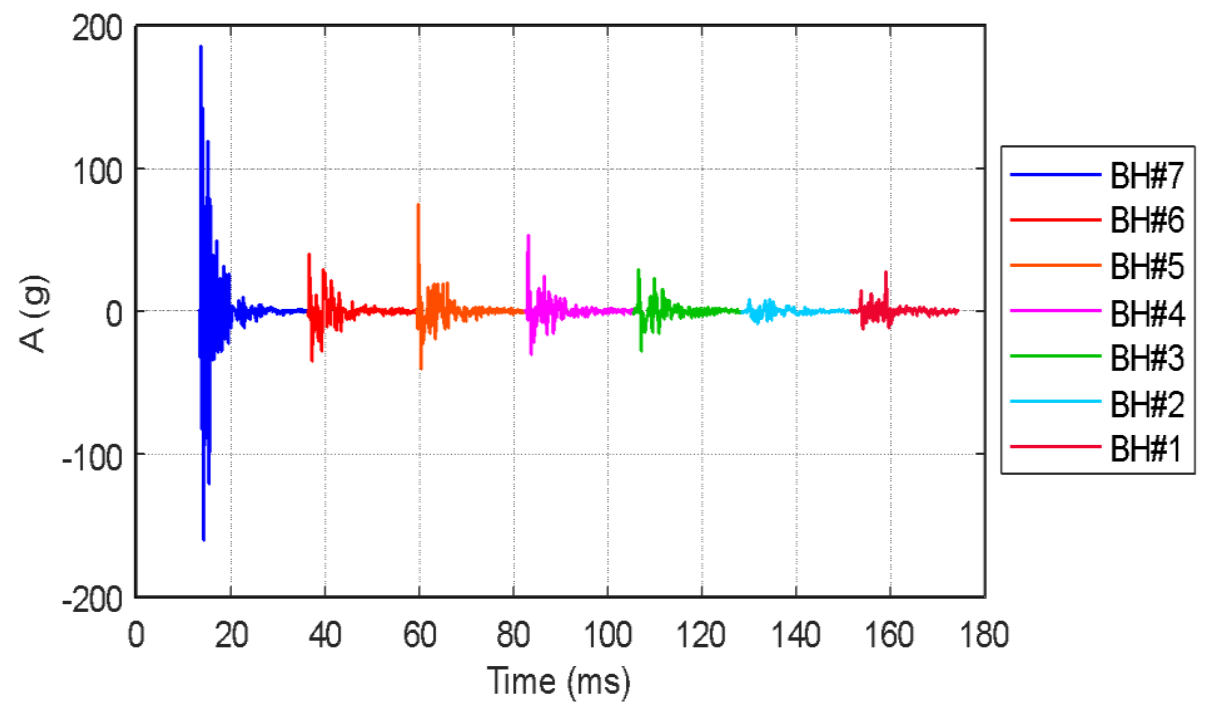

Fig. 4. The signal recorded by A2 in blast B3 (A2B3) passed through the 23 ms-bandwidth rectangular mobile windows.

Once this is done, the processed signals are passed through a zero-phase pass-band Butterworth filter of cut-off frequencies $100 \mathrm{~Hz}$ and $15000 \mathrm{~Hz}$ to suppress noise. This latter frequency is higher than the upper bound of the frequency range in which the acceleration response has a deviation within a $10 \%$. The lower cut-off frequency for the geophone is 30 $\mathrm{Hz}$. The frequency spectra are obtained here using a Fast Fourier Transform algorithm in MATLAB (MATLAB 2020). Figure 5 shows an example of frequency spectra of a signal passed through the rectangular mobile window before and after applying the zero-phase pass-band Butterworth filter. The spectra correspond from top to bottom to the signals from blast holes \#7 (closer to the accelerometer) through \#1; the colours used for the spectra are the same as the corresponding waveforms of Figure 4. 

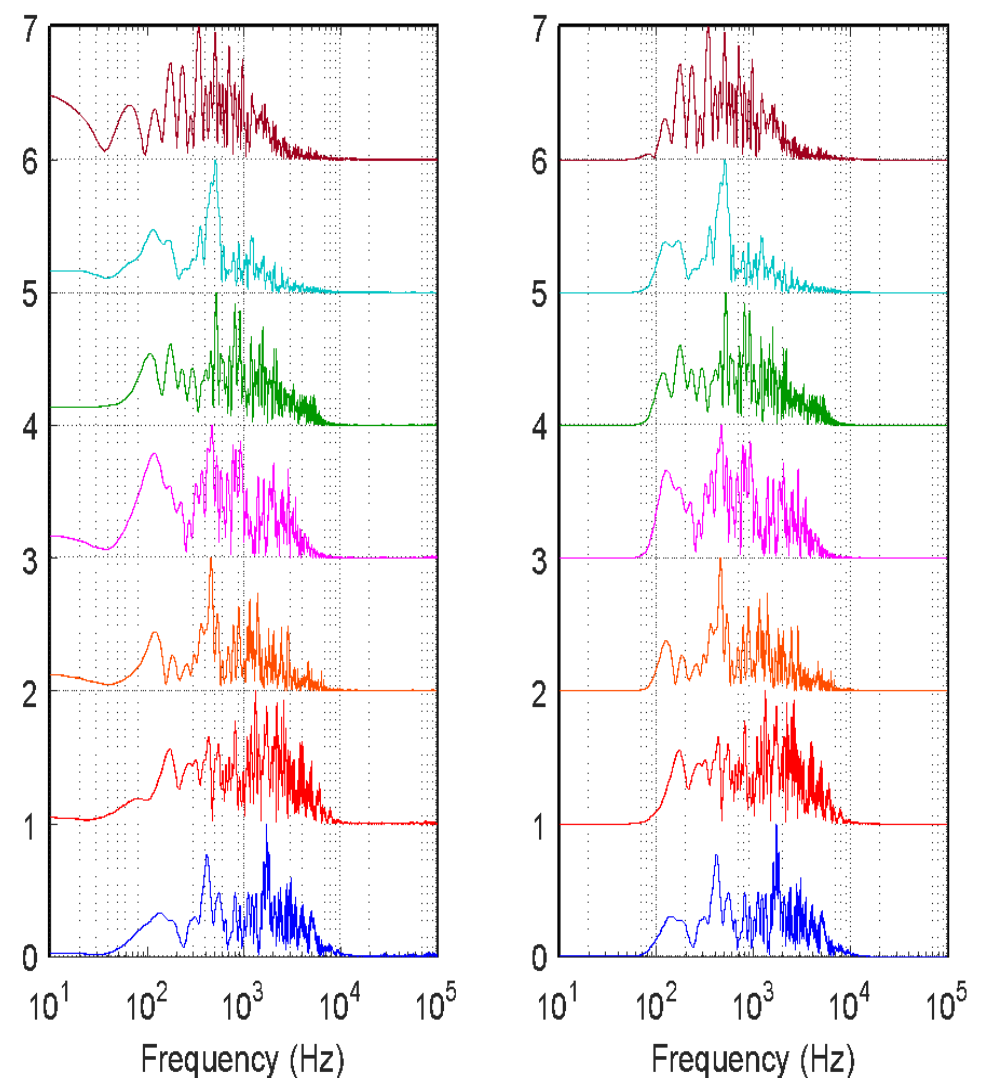

Fig. 5. Individual spectra for A3B9. Raw signal (left), filtered signal (right).

As noted earlier, the parameters chosen to characterize the frequency content of each signal are dominant frequency (DF) and mean frequency (MNF). DF could be defined as the frequency at which the spectrum $P(f)$ reaches its maximum amplitude. MNF could be defined as the "centre" of the power spectrum.

$$
\begin{gathered}
D F=\underset{j}{\arg \max }\left(P_{j}\left(f_{j}\right)\right) \\
M N F=\sum_{j=1}^{m} f_{j} P_{j} / \sum_{j=1}^{m} P_{j}
\end{gathered}
$$

\section{ResUltS AND ANALYSIS}

Once the methodology explained in section 3 is applied,we calculate dominant and mean frequencies from every single spectrum. For the blasts B3 and B7 we calculate an average value between the results of both sensors to avoid duplicities since there are two sensors located practically at the same point.The results obtained are shown in Tables 3 and 4. 
Table 3. Dominant frequencies, DF (Hz) from processed signals / from full-field solution (FFS).

\begin{tabular}{llllllll}
\hline & BH\#1 & BH\#2 & BH\#3 & BH\#4 & BH\#5 & BH\#6 & BH\#7 \\
\hline B3 & $1228 / 1134$ & $1225 / 2610$ & $908 / 2590$ & $1020 / 2468$ & $1375 / 2719$ & $655 / 3080$ & $2528 / 2687$ \\
B6 & $165 / 3289$ & $350 / 2504$ & $145 / 2511$ & $335 / 4184$ & $1515 / 2896$ & $4575 / 5943$ & $9190 / 2838$ \\
B7 & $1668 / 1031$ & $725 / 1108$ & $273 / 1153$ & $542 / 1268$ & $148 / 2421$ & $545 / 3023$ & $3840 / 2645$ \\
B9 & $340 / 1263$ & $508 / 1115$ & $516 / 2653$ & $468 / 2980$ & $456 / 2788$ & $1320 / 2673$ & $1712 / 2794$ \\
A1B9 $^{1}$ & - & - & $532 / 7360$ & $524 / 6582$ & $392 / 7050$ & $388 / 4544$ & $364 / 4511$ \\
G1B9 $^{1}$ & - & - & $64 / 983$ & $48 / 2856$ & $92 / 3746$ & $84 / 1326$ & $64 / 884$ \\
\hline
\end{tabular}

${ }^{1}$ Signals radiated by blastholes \#1 and \#2 were not recorded because the sensors were released from the rock.

Table 4. Mean frequencies, MNF (Hz) from processed signals / from full-field solution (FFS).

\begin{tabular}{llllllll}
\hline & BH\#1 & BH\#2 & BH\#3 & BH\#4 & BH\#5 & BH\#6 & BH\#7 \\
\hline B3 & $1554 / 633$ & $1495 / 639$ & $1774 / 701$ & $2711 / 731$ & $3034 / 790$ & $2240 / 751$ & $3873 / 727$ \\
& 3 & 0 & 4 & 2 & 5 & 4 & 4 \\
B6 & $2425 / 829$ & $1876 / 835$ & $2723 / 877$ & $3326 / 898$ & $3915 / 970$ & $6627 / 998$ & $7688 / 962$ \\
& 1 & 2 & 7 & 8 & 6 & 9 & 9 \\
B7 & $2517 / 614$ & $2297 / 652$ & $2432 / 607$ & $2470 / 654$ & $2201 / 977$ & $3726 / 916$ & $5403 / 825$ \\
& 6 & 1 & 7 & 2 & 1 & 4 & 0 \\
B9 & $2134 / 603$ & $2127 / 640$ & $2534 / 663$ & $2252 / 727$ & $2545 / 814$ & $3161 / 794$ & $4865 / 798$ \\
& 8 & 2 & 0 & 0 & 9 & 5 & 4 \\
A1B9 & - & - & $977 / 9629$ & $1376 / 841$ & $2457 / 871$ & $3648 / 882$ & $8116 / 930$ \\
1 & & & & 2 & 5 & 8 & 2 \\
G1B9 & - & - & $1118 / 528$ & $1922 / 564$ & $2719 / 601$ & $1367 / 564$ & $7830 / 502$ \\
1 & & & 5 & 4 & 7 & 3 & 0 \\
\hline
\end{tabular}

${ }^{1}$ Signals radiated by blast holes \#1 and \#2 were not recorded because the sensors were released from the rock.

Plotting Frequencies in Tables 3-4 against distances in Table 2, we obtain the graphs in Figures 6-7. Two separate analyses have been performed, one on acceleration and the other on velocity. The mean reason for such separation is that frequencies of different seismic fields (e.g. velocity and acceleration) cannot be compared (since higher harmonics are amplified upon differentiation).The model generally overestimates both dominant and mean frequencies for both accelerations and velocities. On average, the mean frequencies obtained with the full-field solution are about 3 times higher than those obtained from raw signals, for both accelerations and velocities. The reason for this is that the propagation model in the full-field solution has been calibrated (especially the $Q$ functions) with amplitudes and no attempt was made to replicate the frequencies. This would probably require some tailoring of the source function and a propagation model that includes scattering.

As expected, there is a slight decrease in dominant frequencies concerning the geometrical distance in signals calculated with FFS in all cases. This may be seen in Figures $6 \mathrm{a}$ and $7 \mathrm{a}$.However, this trend is not so clear in points corresponding to acceleration signals in Figure 6a. Foreman frequencies, a clear negative trend is apparent for both modelled acceleration and measured signals (Figure 6b) and for the modelled waveforms for velocities (Figure 7b), while there is not a clear trend with measured values (Figure 7b). At first glance, the trend could be said to be negative. However, the BH\#7 point $(8.77 \mathrm{~m})$ has a high mean frequency outlier value. An explanation for such a high-frequency value is that measuring in the middle of the blast and so close to it can produce a change in the material state that hides this behaviour; moreover, it is responsible for frequencies of blastholes \#3 and \#7 located at similar distances to be so different. 

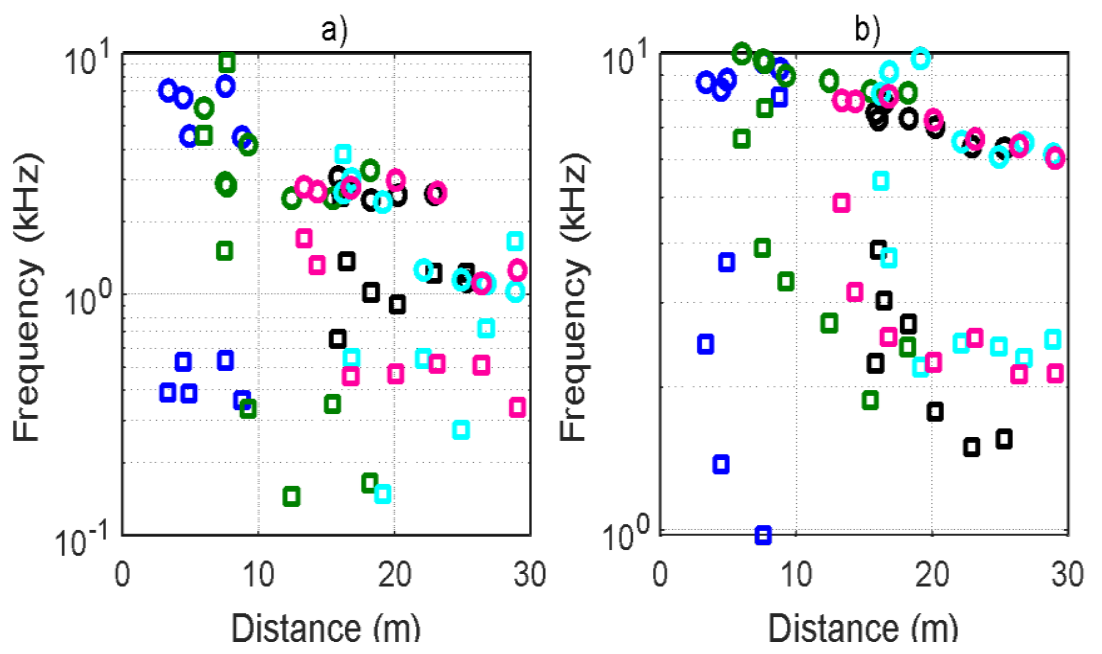

Fig. 6. Dominant frequency, DF (left) and mean frequency MNF (right) vs. geometrical distance for accelerometers. Circles: full-field solution (FFS) waveforms; squares: processed signals. Black: B3,green: B6,light blue: B7, magenta: B9, blue: A1B9.
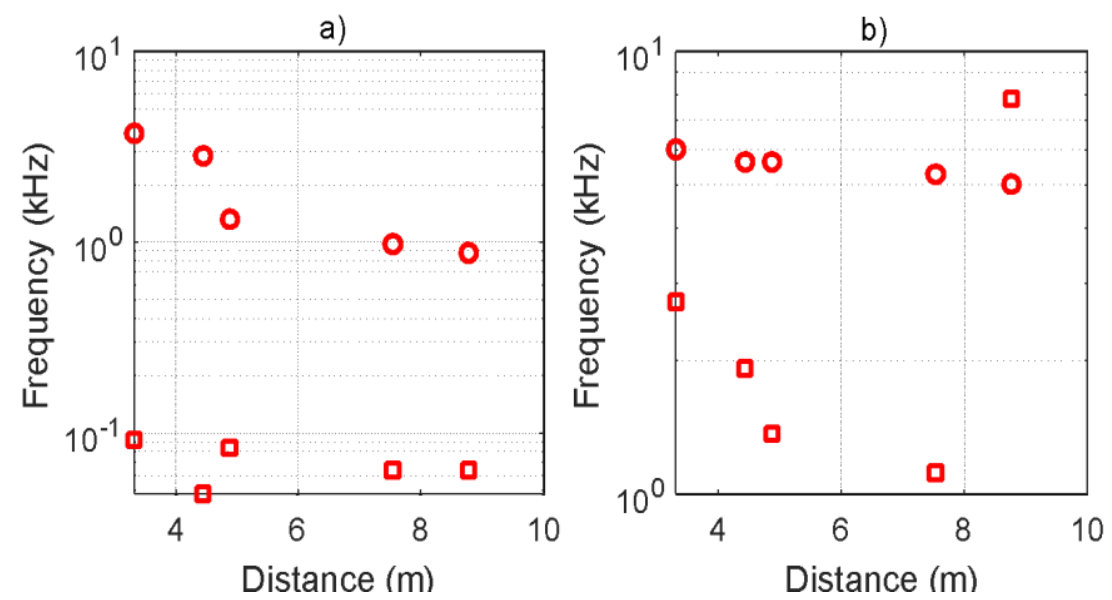

Fig. 7. Dominant frequency, DF (left) and mean frequency, MNF (right) vs geometrical distance for the geophoneG1 in B9. Circles: FFS waveforms; squares: processed.

In order to assess the correlation between frequencies and distances we test the alternative hypothesis that the correlation coefficient is less than zero by applying a left tailed Pearson's linear test using the 'corr.m' MATLAB algorithm (MATLAB, 2020). pvalues less than 0.05 indicate that the correlation coefficient is significantly less than zero. The main statistics of the tests are shown in Tables5 and 6. Correlation coefficients with pvalues lower than $5 \%$ have been bolded in red colour.As tests show in Tables 5 and 6 , fullfield solution attenuates dominant and mean frequencies as geometrical distance increases in velocity and acceleration signals with similar significance level. Moreover, mean frequency significantlyattenuated with distance in processed acceleration signals, which is not the case of dominant frequency. In velocity measured signals, no significant frequency attenuation is observed for processed signals. 
Table 5. Left tailed Pearson's linear correlation tests for accelerations.

\begin{tabular}{lllll}
\hline & \multicolumn{2}{l}{ Dominant frequency } & \multicolumn{2}{l}{ Mean frequency } \\
\hline Accelerations & Model FFS & Proccesed & Model FFS & Processed \\
\hline Correlation coefficient & -0.8382 & -0.2431 & -0.8434 & -0.4276 \\
p-value & $6 \times 10^{-10}$ & 0.0864 & $4 \times 10^{-10}$ & 0.0065 \\
\hline
\end{tabular}

Table 6. Left tailed Pearson's linear correlation tests for velocities.

\begin{tabular}{lllll}
\hline & \multicolumn{2}{l}{ Dominant frequency } & \multicolumn{2}{l}{ Mean frequency } \\
\hline Velocities & Model FFS & Proccesed & Model FFS & Proccesed \\
\hline Correlation coefficient & -0.858 & -0.4118 & -0.9816 & 0.5843 \\
p-value & 0.0314 & 0.2454 & 0.0015 & 0.8496 \\
\hline
\end{tabular}

\section{Conclusions}

This work develops a methodology to obtain the frequency content of seismic signals from blasting using an analytical vibration prediction model. It has been proven that the full-field solution attenuates the frequency content with distance, making it a valid tool for frequency analysis. This has two important advantages over the traditional empirical methods: (i) complete waveforms are obtained whereas with traditional methods it is not possible and (ii) both velocities and accelerations may be used in the same analysis, since we may predict both seismic fields at the same point whereas with empirical methods an attenuation law in frequency is often calibrated by using only one of them (usually velocity). This allows a mining operation to use both geophones and accelerometers, equally.

As expected, the mean frequencies of individual signals coming from each hole shift to lower frequencies as the distance to the sensor increases. The results also show that the fullfield solution overestimates the dominant and mean frequencies in the entire frequency domain when compared to the measured signals. This may be explained as follows: (i) the model does not allow for modelling free surfaces or discontinuities in the rock mass, such as fractures, voids, faults, etc.; this is a difficult, if not impossible, problem to solve analytically and it plays in favour of numerical models; (ii) the attenuation $Q$-model does not consider the effects of elastic scattering, that may be significantly higher than viscoelastic attenuation; (iii) the source function may significantly influence the frequency content in a way that a source function with high average frequency causes seismic signals with high-frequency content.

The results from statistical tests show a better correlation with distance for mean frequency values than for dominant frequencies, especially in the case of velocity signals obtained with FFS. Such correlation has only been obtained for acceleration processed signals using mean frequencies instead of dominant ones. This confirms the initial hypothesis that mean frequency is a better indicator of the frequency content of seismic signals. In view of this, a revision of the standards would be advisable to incorporate procedures that include mean frequency to characterize the frequency content of a signal from blasting.

\section{REFERENCES}

1. AENOR-Asociación Española de Normalización y Certificación. Control de vibracionesproducidasporvoladuras. Norma UNE 22-381-93. Madrid(1993).

2. Blair, D. Geophys J Int. Estimates of seismic attenuation using vibrational resonance and pulse transmission in four large blocks of rock126 (1), 135-146 (1996). 
3. Blair, D. Geophysics. A comparison of Heelan and exact solutions for seismic radiation from a short cylindrical charge 72(2) (2007).

4. Blair, D. GeophysicsSeismic radiation from an explosive column75(1) (2010).

5. Blair, D. Int J Rock Mech Min Sci. Blast vibration dependence on charge length, velocity of detonation and layered media, 65, 29-39 (2014).

6. Castedo, R. Natale, M., López, L. M., Sanchidrián, J.A., Santos, A.P., Navarro J. \&Segarra, P. Int J Rock Mech Min Sci. Estimation of Jones-Wilkins-Lee parameters of emulsion explosives using cylinder tests and their numerical validation112, 290-301 (2018).

7. Gómez S, Bermejo M, Navarro J. European Union's Horizon 2020 Research and Innovation Program, Gran AgreementNear-Field Seismic Model for Rock Damage. SLIM Deliverable Report D3.1,Madrid (2019).

8. Gómez, S. Sanchidrián, J. A. \&Segarra, P. International Journal of Rock Mechanics and Mining SciencesNear-field vibration from blasting and rock damage prediction with a full-field solution 134 (2020).

9. Gou, Y. Shi, X. Zhou, J. Qiu, X. Chen, X., Huo, X. International Journal of Rock Mechanics and Mining Sciences Attenuation assessment of blast-induced virations derived from an underground mine 127 (2020).

10. Hernández-Enrile, J. L. Evoluciónmicroestructural de rocascuarzofeldespáticascomoresultado del aumento de la deformación en la milonita de Toledo (1981).

11. Holmberg, R. \&Persson, P-A. The Swedish Approach to Contour Blasting. SveDeFo (1978).

12. Hustrulid, W. \& Lu, W. Explosives and Blasting Technique The Lu-Hustrulid approach for calculating the peak particle velocity caused by blasting, 291-300 (2003).

13. Iverson, S. Kerkering, C., Hustrulid, W. NatlInstOccupSaf HealApplication of the NIOSH-Modified Holmberg-Persson Approach to Perimeter Blast Design(2008).

14. Kjartansson, E. J Geophys Res Solid Earth Constant Q-wave propagation and attenuation 84 (1978).

15. Martínez-Salanova, J. Martín, L. Gutiérrez, G. Martín, D. Sánchez, R. Andonaegui, P., Iglesias, A. Memoria de la Hoja n ${ }^{\circ} 658$ (Mora). MapaGeológico de España E. 1:50.000 (MAGNA), SegundaSerie, Primeraedición. Madrid (Spain): IGME (2009).

16. Meredith, J. A. Numerical and Analytical Modelling of Downhole Seismic Sources: The Near and Far Field. (Doctoral dissertation, Massachusetts Institute of Technology) (1990).

17. Norma UNE 22-381-93. Control de vibracionesproducidasporlasvoladuras, AENOR, Madrid (1993).

18. MATLAB. version 9.8.0.1323502 (R2010a). Natick, Massachusetts: The MathWorks Inc. (2020).

19. Quan, Y. \& Harris, J. M. Geophysics Seismic attenuation tomography using the frequency shift method62(3), 895-905 (1997).

20. Tubman, K. M. Cheng, C. H. \& Toksöz, M. N. GeophysicsSynthetic full waveform acoustic logs in cased boreholes49(7), 1051-1059 (1984). 